\title{
RECURRENT MESALAZINE-INDUCED MYOPERICARDITIS IN A PATIENT WITH ULCERATIVE COLITIS
}

\author{
EUN HYE PARK, MD ${ }^{1}$, BYUNG JIN KIM, MD ${ }^{1}$, JUNG KWON HUH, MD², EUN HAENG JEONG, MD ${ }^{1}$, \\ SANG HYUK LEE, MD ${ }^{1}$, KI BAE BANG, MD¹, JI SOO SEOL, MD¹, JOO WOOK SUNG, MD¹, \\ BUM SOO KIM, MD ${ }^{1}$ AND JIN HO KANG, MD ${ }^{1}$ \\ 'DIVISION OF CARDIOLOGY, DEPARTMENT OF INTERNAL MEDICINE, KANGBUK SAMSUNG HOSPITAL, SUNGKYUNKWAN UNIVERSITY \\ SCHOOL OF MEDICINE, SEOUL, KOREA \\ ${ }^{2}$ DEPARTMENT OF INTERNAL MEDICINE, HANA GENERAL HOSPITAL, CHEONGJU, KOREA
}

Inflammatory bowel disease (IBD) is considered as a dysregulated immune mediated disease. Pericarditis in IBD is a very rare disease both as an extra-intestinal manifestation of IBD and an adverse reaction of therapeutic drug for IBD such as mesalazine or sulfasalazine. A 26-year-old IBD male patient who had been taking mesalazine regularly for about 1 month was referred to our hospital because of fever, chest discomfort, and abnormal electrocardiographic findings. The patients was diagnosed as acute myopericarditis, and recovered after cessation of mesalazine using steroid and aspirin. When mesalazine was re-medicated some days after discharge, he suffered from myopericarditis again. Subsequently, myopericarditis was resolved just after cessation of mesalazine again. These findings suggest that the development of myopericarditis is caused by mesalazine.

KEY WORDS: Myopericarditis · Mesalazine - Inflammatory bowel disease.

\section{INTRODUCTION}

Pericarditis is rarely reported in inflammatory bowel disease, which mostly arises as an extra-intestinal manifestation but it can infrequently appear as an adverse effect of therapeutic drugs for inflammatory bowel disease (IBD) including mesalazine. ${ }^{1-3)}$ The prevalence of mesalazine-induced pericarditis is very low. ${ }^{4}$ Here, we report a very rare case of 26-year-old IBD male patient who presented mesalazine-related recurrent myopericarditis.

\section{CASE}

The patient was 26-year-old man who was diagnosed as ulcerative colitis (UC) at other hospital 6 years ago. Since then, he had been taking medication irregularly by himself. One month ago, he received surgical treatment for anal fistula and started to take mesalazine-containing regimen (Asacol $2.4 \mathrm{~g}$ per day) regularly. He did not feel uncomfortable while taking medication for about 1 month. At 3 days before admission, he intermittently felt febrile sensation and anterior chest discomfort on resting. The chest discomfort was aggravated by positional change and deep breathing but relieved by stable supine position. He was admitted to our hospital via emergency room to evaluate his symptoms.

At the time of admission, body temperature was $38.3^{\circ} \mathrm{C}$, systolic/diastolic blood pressure was $100 / 60 \mathrm{mmHg}$, heart rate was 124 beats/min and respiratory rate was 20 times/min. Laboratory tests including serum liver enzymes, serum blood urea nitrogen/creatinine levels, serum cardiac enzyme levels were within normal range, however, the $\mathrm{N}$-terminal pro-brain natriuretic peptide (NT-proBNP) level was increased to 1877 $\mathrm{pg} / \mathrm{mL}$ (reference value: 0-349 $\mathrm{pg} / \mathrm{mL}$ ). White blood cell count was $10900 / \mathrm{mm}^{3}$ (reference value: $4100-10200 / \mathrm{mm}^{3}$ ), neutrophil count was $9232 / \mathrm{mm}^{3}(84.7 \%$, reference value: $38.3-69.3 \%)$ and eosinophil count was $32 / \mathrm{mm}^{3}(0.3 \%$, reference value: $0.7-8.1 \%)$. Hemoglobin level was slightly low $(10.4 \mathrm{~g} / \mathrm{dL})$. The erythrocyte sedimentation rate (ESR) (refer-

- Received: June 28, 2012 -Revised: August 6, 2012 -Accepted: August 8, 2012

- Address for Correspondence: Byung Jin Kim, Division of Cardiology, Department of Internal Medicine, Kangbuk Samsung Hospital, Sungkyunkwan University

School of Medicine, 29 Saemunan-ro, Jongno-gu, Seoul 110-746, Korea Tel: +82-2-2001-2401, Fax: +82-2-2001-2400, E-mail: bjjake.kim@samsung.com

- This is an Open Access article distributed under the terms of the Creative Commons Attribution Non-Commercial License (http://creativecommons.org/licenses/by-nc/3.0)

which permits unrestricted non-commercial use, distribution, and reproduction in any medium, provided the original work is properly cited. 
ence value: $2-30 \mathrm{~mm} / \mathrm{hr}$ ) and C-reactive protein (CRP) (reference value: $\leq 0.59 \mathrm{mg} / \mathrm{dL}$ ) were elevated up to $93 \mathrm{~mm} / \mathrm{hr}$ and $15 \mathrm{mg} / \mathrm{dL}$, respectively. The electrocardiogram (ECG) revealed distinct ST segment elevations in leads I, II, aVL, and $\mathrm{V}_{2-6}$ (Fig. 1). Echocardiogram showed mild pericardial effusion (about $0.2 \mathrm{~cm}$ ) in the posterior space and slightly increased pericardial thickness, and global hypokinesis of the left ventricle (Fig. 2). Left ventricular ejection fraction was $60.3 \%$ and left ventricular internal dimension at diastole was $4.73 \mathrm{~cm}$.

He was diagnosed as acute myopericarditis. After taking oral aspirin of $2 \mathrm{~g}$ per day and prednisolone of $40 \mathrm{mg}$ per day with cessation of mesalazine medication, he felt no more chest pain and fever was disappeared at 3 days after medical treatment. The abnormal ECG findings were gradually normalized within 7 days. On echocardiogram at 11 days after treatment, pericardial effusion, increased pericardial thickness, and wall motion abnormality were completely improved. Finally, he was discharged with aspirin $2 \mathrm{~g}$ per day and prednisolone $20 \mathrm{mg}$ per day. Unfortunately, the mesalazine ( $3 \mathrm{~g}$ per day) was re-administered at 6 days after discharge by his gastroenterologist. At 3 days after mesalazine re-medication, he felt chest pain and febrile sensation again, although he had been taking aspirin ( $2 \mathrm{~g}$ per day) and prednisolone (20 $\mathrm{mg}$ per day) at that time. He was readmitted to our hospital, and the echocardiographic findings showed minimal pericardial effusion and mild global hypokinesia of the left ventricle again, although the findings were milder than those at the time of first admission. Acute phase reactants were also elevated without leukocytosis nor eosinophilia (ESR $88 \mathrm{~mm} / \mathrm{hr}$, CRP $18.30 \mathrm{mg} / \mathrm{dL}$, white blood cell count $6590 / \mathrm{mm}^{3}$ and eosinophil fraction $8 \%$ ). Mesalazine was immediately stopped with maintaining other

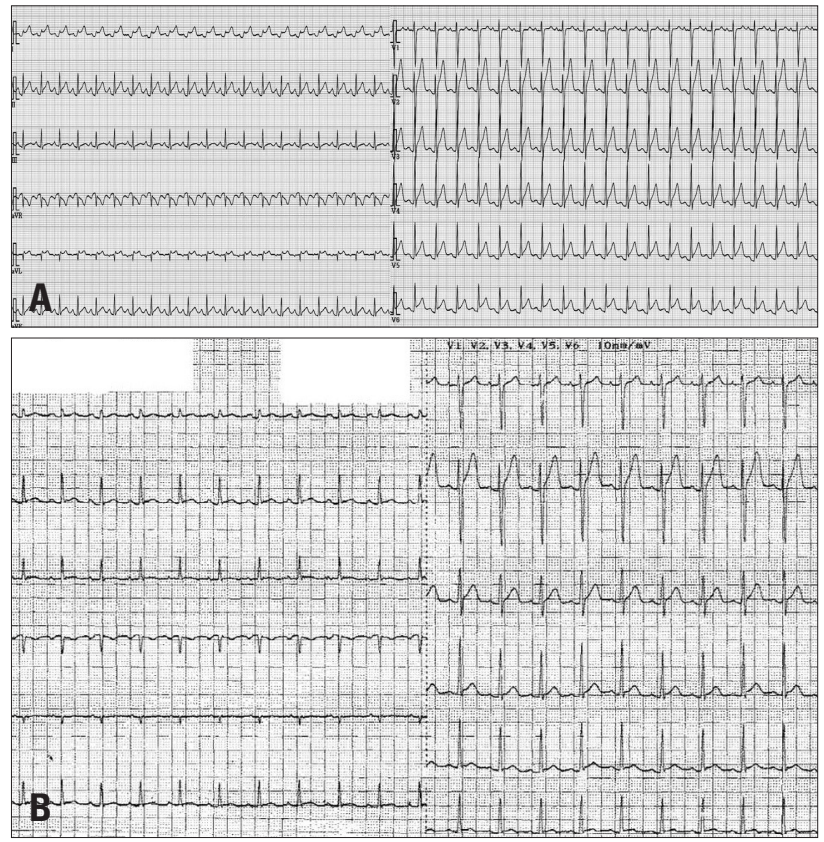

Fig. 1. Electrocardiographic findings on first admission. This ECG was recorded with time scale of $25 \mathrm{~mm} / \mathrm{s}$ on the horizontal axis and a voltage sensitivity of $10 \mathrm{~mm} / \mathrm{mV}$ on the vertical axis. A: ST segment elevation on lead I, II, aVL, V2-6 without reciprocal change at first admission. B: Improved ST segment elevation at discharge. ECG: electrocardiogram.
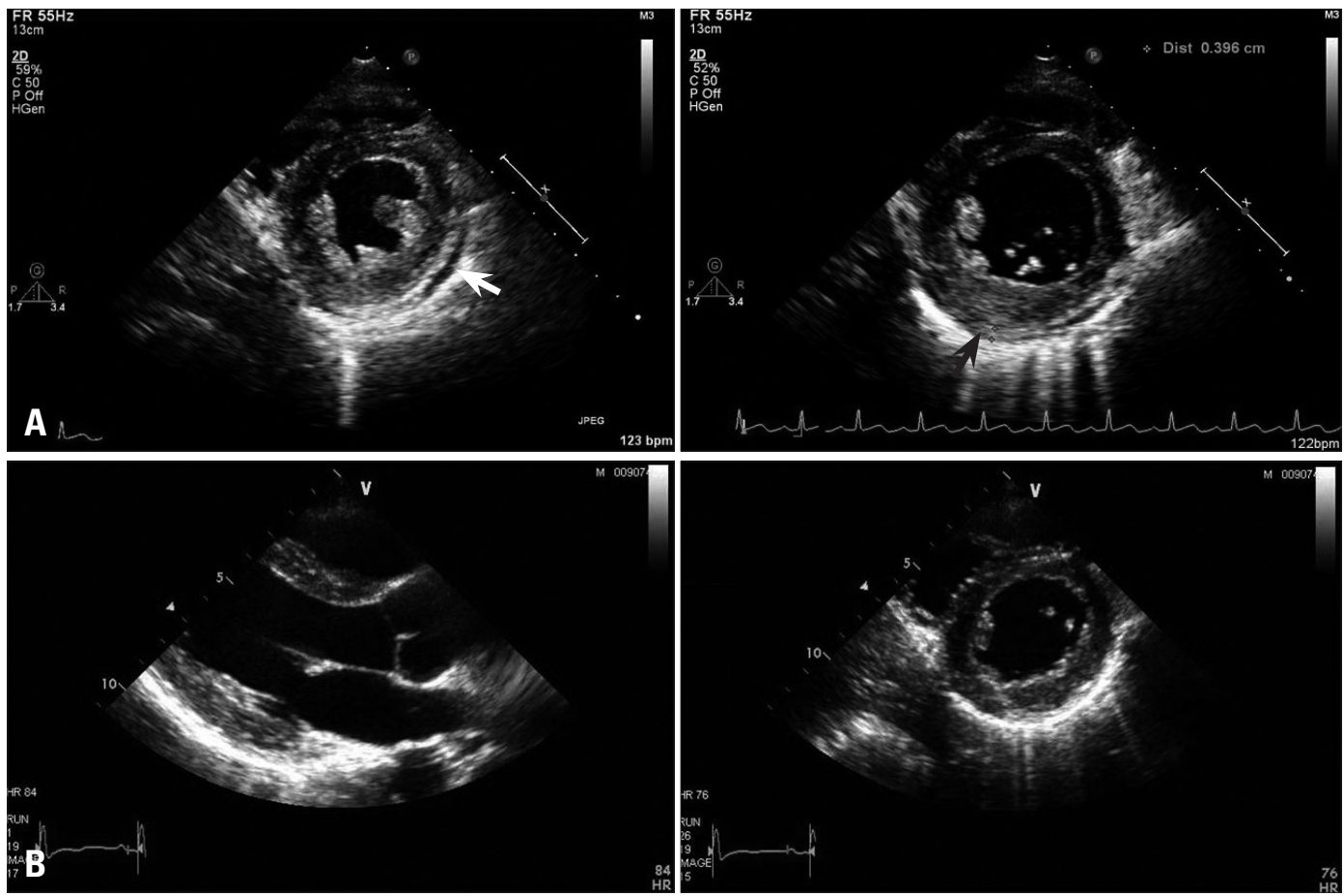

Fig. 2. Echocardiographic findings on first admission. A: Minimal pericardial effusion (white arrow) and increased posterolateral pericardial thickness about $0.39 \mathrm{~cm}$ (black arrow). B: Improvement of pericardial effusion and increased pericardial thickness at discharge. 
medications including aspirin and prednisolone. After then, he showed dramatic improvement of chest pain and fever within 3 days after cessation of mesalazine. There was no abnormal arrhythmic rhythm change in ECG monitor except intermittent ventricular premature beats during admission. When discharge, we recommended not to prescribe mesalazine for him ever again to his doctor. However, we have no more information on the patient because he did not return for any treatment ever since.

\section{DISCUSSION}

In our case, the patient was 26-year-old male who had been treated with mesalazine containing oral medication irregularly for UC but he was diagnosed as acute myopericarditis on 1 month after regular mesalazine oral intake. Subsequently, recurrent myopericarditis was developed by re-medication of mesalazine.

The prevalence of acute pericarditis as an extra-intestinal manifestation is known as $0.23 \%$ in UC patients, $0.19 \%$ in Crohn's disease patients and it may be developed independently regardless of disease activity. ${ }^{24) 55}$ The incidence of mesalazine-induced pericarditis is probably very low and it mostly occurs within a few weeks after mesalazine medication. ${ }^{6}$

Although the precise pathophysiology of mesalazine-induced pericarditis is poorly understood, it has been known that mesalazine-induced pericarditis may be attributed to an acute hypersensitivity reaction against 5 -aminosalicylic acid (5-ASA) regimen. ${ }^{26) 77}$ One report showed increased proliferation index of serial lymphocyte stimulation test, which means T-cell mediated type IV drug hypersensitivity reaction. ${ }^{8)}$

Several cases of recurrent mesalazine-induced pericarditis have been reported; among them, a case reported recurrent pericarditis developed with changing mesalazine administration route from oral to anal suppositories type in an UC patient; ${ }^{1)}$ another case described recurrent pericarditis occurred at 6 hours after re-medication of very low dose 5-ASA in an UC patient. ${ }^{2}$ These cases suggest that mesalazine induced pericarditis can be recurred with mesalazine medication, irrespective of mesalazine administration route and doses. There was a fatal case with mesalazine-associated myocarditis which was developed about 13 days after mesalazine medication, and he (or she) died by ventricular fibrillation with cardiogenic shock. ${ }^{9)}$

The specific view in our case is that the mesalazine-induced myopericarditis was developed about 1 month after regular mesalazine intake, although he already had been taking it irregularly. It means that its development may be unrelated to duration of mesalazine medication. In our case, the first event of myopericarditis may be the result of idiosyncratic reaction or hypersensitivity reaction of mesalazine because he had already been taken mesalazine irregularly for about 6 years. However, the putative mechanism of its second events could be attributable to hypersensitivity reaction of mesalazine, considering that it occurred right after mesalazine re-medication and it disappeared with cessation of mesalazine.

In conclusion, we present a case of mesalazine-induced myopericarditis that was successfully improved by discontinuation of the medication. Because mesalazine-induced myopericarditis is very rare but could be fatal, meticulous attention for the accurate diagnosis of pericarditis in IBD patients taking mesalazine would be required if the myopericarditis is caused by extra-intestinal manifestation of IBD or mesalazineinduced hypersensitivity.

\section{REFERENCES}

1. Bernal-Sprekelsen JC, de las Marinas MD, Salvador A, Landete FJ, Morera FJ. Recurrent pericarditis in a patient with ulcerative proctitis due to mesalazine suppositories. Int J Colorectal Dis 2010;25:1143-4.

2. Ishikawa N, Imamura T, Nakajima K, Yamaga J, Yuchi H, Ootsuka M, Inatsu H, Aoki T, Eto T. Acute pericarditis associated with 5-aminosalicylic acid (5-ASA) treatment for severe active ulcerative colitis. Intern Med 2001;40:901-4.

3. García-Morán S, Sáez-Royuela F, Pérez-Alvarez JC, Gento E, Téllez J. Myopericarditis and mitral insuffciency associated with ulcerative colitis treated with mesalazine. Inflamm Bowel Dis 2006;12:334-5.

4. Vayre F, Vayre-Oundjian L, Monsuez JJ. Pericarditis associated with longstanding mesalazine administration in a patient. Int J Cardiol 1999; 68:243-5.

5. Bernstein CN, Wajda A, Blanchard JF. The clustering of other chronic inflammatory diseases in inflammatory bowel disease: a population-based study. Gastroenterology 2005;129:827-36.

6. Sentongo TA, Piccoli DA. Recurrent pericarditis due to mesalamine hypersensitivity: a pediatric case report and review of the literature. J Pediatr Gastroenterol Nutr 1998;27:344-7.

7. Perrot S, Aslangul E, Szwebel T, Gadhoum H, Romnicianu S, Le Jeunne C. Sulfasalazine-induced pericarditis in a patient with ulcerative colitis without recurrence when switching to mesalazine. Int $J$ Colorectal Dis 2007:22:1119-21.

8. Jenss H, Becker EW, Weber P. Pericardial effusion during treatment with 5-aminosalicylic acid in a patient with Crobn's disease. Am J Gastroenterol 1990;85:332-3.

9. Kristensen KS, Høegholm A, Bohr L, Friis S. Fatal myocarditis associated with mesalazine. Lancet 1990;335:605. 